\section{Commentary: Aspects of epidermal growth factor receptor status: Prognostic factor versus predictive factor}

\author{
Morihito Okada, MD, $\mathrm{PhD}$
}

Although "prognostic factor" and "predictive factor" are frequently applied in an interchangeable manner and are often left undefined, these factors have been used in many articles to define the association between clinical results and biomarkers. "Prognostic factor" is a measure that is related to clinical results without any therapy or with the application of a standard therapy that patients are likely to get and may be considered as that of the natural history of the disease. "Predictive factor" is a measure that is related to the presence or absence of a response to a particular therapy, including molecularly targeted therapy, and indicates a differential merit from the therapy that relies on the status of the biomarker.

The important article by Deng and colleagues ${ }^{1}$ published in this issue of the Journal describes the influence of a mutation in the epidermal growth factor receptor (EGFR) gene on the prognosis of patients with resected lung adenocarcinoma. They found that the EGFR mutation was a poor prognostic factor in patients with radiologic solid, histologic acinar pattern-predominant adenocarcinoma/ papillary pattern-predominant adenocarcinoma/invasive mucinous adenocarcinoma, and pathologic stage II and III diseases. Recurrence-free survival and overall survival curves between patients with the EGFR mutation and patients with the wild-type were reversed. The status of the EGFR mutation, which confers risk of recurrence, and renders it a factor that is associated with a good prognosis from the viewpoint of overall survival, is probably based on the influence of administering an EGFR tyrosine kinase inhibitor (TKI) after a disease has recurred.

From the Department of Surgical Oncology, Hiroshima University, Hiroshima, Japan. Disclosures: The author reported no conflicts of interest.

The Journal policy requires editors and reviewers to disclose conflicts of interest and to decline handling or reviewing manuscripts for which they may have a conflict of interest. The editors and reviewers of this article have no conflicts of interest.

Received for publication July 28, 2020; revisions received July 28, 2020; accepted for publication July 29, 2020; available ahead of print Aug 3, 2020.

Address for reprints: Morihito Okada, MD, PhD, 1-2-3 Kasumi, Minami-ku, Hiroshima, 734-0037 Japan (E-mail: morihito@ hiroshima-u.ac.jp).

J Thorac Cardiovasc Surg 2021;162:678-9

$0022-5223 / \$ 36.00$

Copyright (c) 2020 by The American Association for Thoracic Surgery

https://doi.org/10.1016/j.jtcvs.2020.07.085

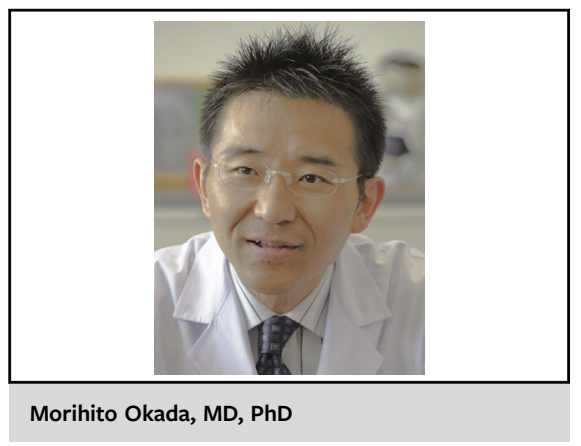

CENTRAL MESSAGE

An EGFR mutation can be a positive prognostic factor using EGFR-TKI, which is a powerful predictor of a therapeutic effect; however, it might be a negative prognostic factor without EGFRTKI.

Disease-free survival results of the randomized phase III ADAURA trial unequivocally support the use of osimertinib, a third-generation EGFR-TKI approved for use in patients with metastatic non-small cell lung cancer, as a standard component of care in patients with EGFR-mutated non-small cell lung cancer after complete tumor resection from the American Society of Clinical Oncology 2020 Virtual Scientific Program Plenary Session as reported by $\mathrm{Wu}$ and colleagues. ${ }^{2}$ Outcomes from an unplanned interim analysis demonstrated an $83 \%$ reduction in the risk of recurrence or death with adjuvant osimertinib in comparison with placebo among patients with stage II or IIIA disease (hazard ratio, $0.17 ; 95 \%$ confidence interval, $0.12-0.23 ; P<.0001$ ), and these findings remained consistent when evaluating all 682 patients in the trial, which included individuals with stage IB disease (hazard ratio, $0.21 ; 95 \%$ confidence interval, 0.16-0.28; $P<.0001)$. When osimertinib was routinely used in the setting of adjuvant therapy, recurrence-free survival was considered to be better among patients with an EGFR mutation compared with the wild-type.

Thus, EGFR-mutated status can be a good "prognostic factor" for patients treated with EGFR-TKI because it is a powerful "predictive factor" of a therapeutic effect, even though the EGFR mutation per se might be a poor "prognostic factor" for patients with lung adenocarcinoma who are not treated with EGFR-TKI. 


\section{References}

1. Deng C, Zhang Y, Ma Z, Fu F, Deng L, Li Y, et al. Prognostic value of epidermal growth factor receptor gene mutations in resected lung adenocarcinoma. $J$ Thorac Cardiovasc Surg. 2021;162:664-74.e7.
2. Wu YL, Herbst RS, Mann H, Rukazenkov Y, Marotti M, Tsuboi M. ADAURA phase III, double-blind, randomized study of osimertinib versus placebo in EGFR mutation-positive early-stage NSCLC after complete surgical resection. Clin Lung Cancer. 2018;19:e533-6. 